\title{
Comparing personal and social optimism biases: magnitude, overlap, modifiability, and links with social identification and expertise
}

\author{
Tatjana Aue ${ }^{1 凶}$, Mihai Dricu ${ }^{1}$, Dominik A. Moser ${ }^{1}$, Boris Mayer ${ }^{1} \&$ Stephanie Bührer ${ }^{1}$
}

Individuals are more optimistic about their own future than a comparable person's future (personal optimism bias). In addition, they show overoptimism toward people or social groups they identify with compared with those they do not identify with (social optimism bias). However, commonalities and differences between personal and social forms of optimism bias remain to be addressed. Data from an experiment on anticipated performances in soccer (including 160 participants), revealed (a) comparable magnitudes of personal and social optimism biases, and (b) only partial overlap between personal and social optimism biases. We further found the magnitude of the biases to depend on (c) prior experience in the investigated area. Social optimism bias, however, did not correlate with (d) the extent to which the participants identified with a social in-group. In addition, we demonstrate that (e) despite the availability of objective feedback, both personal and social optimism biases are hard to overcome. Our data further suggest ( $f$ ) the existence of qualitatively different social optimism biases; biases that can possibly be distinguished by their degree of automaticity or the adoption of a more affective vs. utilitarian stance. Consequently, the present research reveals that the phenomenon of social optimism bias needs further refinement to adequately address its specific sub-components.

\footnotetext{
${ }^{1}$ Institute of Psychology, University of Bern, Bern, Switzerland. ${ }_{\text {email: tatjana.aue@unibe.ch }}$
} 
positive view of the world is associated with the experience of positive emotions, sociability, as well as mental and physical health (Fox, 2012; Garrett et al., 2014; McNally, 1995). Optimistic rather than pessimistic expectancies regarding the future contribute to these effects in important ways (Scheier and Carver, 1992; Seligman and Csikszentmihalyi, 2000). While most of the time advantageous, optimistic human expectancies often surpass the level justified by a rational consideration of the information at hand (Weinstein, 1980; Windschitl and Stuart, 2015): When asked about their future, most people are overly optimistic - with the probability of desirable future events (e.g., having success in one's job) being overestimated and the probability of undesirable future events (e.g., incurring cancer) being underestimated. This phenomenon has hence been termed optimism bias (alternatively: unrealistic optimism, comparative optimism, wishful thinking, or desirability bias; e.g., Dricu et al., 2020; Krizan and Windschitl, 2007) and describes irrational confidence in desirable outcomes (e.g., Hollander, 2004; Lench and Ditto, 2008; McGuire, 1960; Weinstein, 1980).

Optimism bias appears in different domains, including individualistic settings such as personal health (Weinstein, 1980), own profession (Hoch, 1985), and personal gambling activities (Kress et al., 2018; Lench and Ditto, 2008). It also arises in social group settings such as sports (Aue et al., 2012; Babad, 1987; Love et al., 2015) and politics (Babad, 1997; Hollander, 2004). Interestingly, people have an overly optimistic outlook regarding future events not only for themselves (e.g., McGuire, 1960; Sharot et al., 2011), but also for the social groups that they identify with (so-called in-groups; Aue et al., 2012; Dricu et al., 2018; Hollander, 2004; Love et al., 2015; Simmons and Massey, 2012) or empathize with (e.g., the elderly; Dricu et al., 2018). At the same time, people display a pessimistic outlook regarding future events for disliked groups that they do not identify with (Dricu et al., 2018).

Along the lines of social identity theory (Abrams and Hogg, 2004; Tajfel and Turner, 1986), the social optimism bias may arise in order to preserve the positive image of the in-group andthrough identification-positive self-esteem (cf. Hogg and Abrams, 1990; Tajfel et al., 1971; Turner and Onorato, 1999). Correspondingly, both personal and social optimism biases are supposed to serve adaptive purposes (Aue et al., 2012; Granberg and Holmberg, 1988; Shepperd et al., 2015) and contribute to mental health (Korn et al., 2014; Zetsche et al., 2015). However, to date, personal and social biases have not been explicitly compared. Consequently, similarities and divergences between personal and social forms of optimism bias remain to be identified.

The current study pursued multiple aims. First, we examined the degree to which personal optimism bias and different forms of social optimism bias are comparable in magnitude (Aim 1: Quantification of personal and social forms of optimism bias). Social optimism bias can be divided into subtypes that vary in terms of relevance to the individual. An individual may, for instance, be overoptimistic for teammates in sports (indirect social optimism bias). While the individual is not personally involved in such anticipated scenarios, good performance of the teammate would increase the standing of the entire team, thereby having an indirect influence on the individual's own future possibilities. Additionally, an individual may be over optimistic for others (e.g., favorite international soccer team) whose performance is only abstractly relevant to the individual because their performance will not impact his or her personal future (abstract social optimism bias).

While all three biases are supposed to exert feedback effects on an individual's self-esteem (Abrams and Hogg, 2010), personal optimism bias may be the most direct means to increase self-esteem. Therefore, personal optimism bias might be expected to be characterized by a greater magnitude than are social forms of optimism bias. On the other hand, in-group successes and failures have also been shown to exert powerful effects on the individual self-concept, and those effects resemble the effects arising from personal achievements (Abrams and Hogg, 2004; Boen et al., 2002; Cialdini et al., 1976; Tajfel and Turner, 1986). Comparing the magnitudes of the personal, indirect social, and abstract social biases may be a first step toward assessing the relative contributions of these biases to individual well-being.

Second, we tested the overlap between the three different biases by looking at their correlation (Aim 2: Overlap between personal and social forms of optimism bias). Expressed differently, we verified whether those participants who engaged strongly in personal optimism bias were identical to those who displayed powerful social optimism biases. This aim thus tested for general vs. specific ease of engaging in the different optimistic biases (no directed hypothesis). Strong associations between the biases would be in support of the existence of comparable underlying neurocognitive processes. As such, attempts at increasing self-esteem, for instance, might go along with an exchangeable engagement of all three biases. Low correlations, on the contrary, would speak to qualitatively different basic neurocognitive mechanisms. Alternatively, individuals may preferably engage in one optimism bias or the other to attain/sustain positive emotionality.

Third, research on optimism bias has shown an influence of prior experience with a situation (e.g., Grezo, 2017; HelwegLarsen and Shepperd, 2001; Weinstein, 1980). Generally, it has been observed that personal optimism bias decreases as experience with (or knowledge about) an event increases (Barnoy et al., 2003; Campbell et al., 2007; Cho et al., 2010). Among other explanations, this effect was attributed to changes in perceived control over the event's occurrence. While these observations have been made in the personal domain, corresponding effects in the social domain await to be addressed (Aim 3: Influence of expertise on personal and social forms of optimism bias). Based on results in the area of personal optimism bias, we hypothesized that the magnitude of the biases would be overall reduced in experts.

Fourth, behavioral data show that people project self-attributes to individuals whom they perceive to be close or similar to themselves but use stereotypes for individuals whom they perceive as dissimilar to themselves (Ames, 2004; Dricu et al., 2018). Another question that arises is, hence, whether people are overly optimistic about their own social group because they adopt the perspective "as if it were me" and then process information in the same way as for the self (Aim 4: Influence of social identification and experienced similarities on social forms of optimism bias). Therefore, we tested whether the extent of biased social expectancies depends on the strength of identification with the ingroup. We predicted that social biases vary as a positive function of the perceived similarity between the self, on the one hand, and the indirect and abstract in-groups, on the other hand (for a positive association between social identification and in-group favoritism, see also Verkuyten, 2007).

Fifth, recent findings have shown that optimistic biases in the personal domain are hard to overcome (e.g., Chowdhury et al., 2014; Kuzmanovic et al., 2015, 2016; Marks and Baines, 2017; Sharot et al., 2011; Weinstein and Klein, 1995). Comparably, in the area of social optimism bias, it has been demonstrated that monetary incentives for accurate predictions and the availability of objective background information do not eliminate the bias (Aue et al., 2012; Babad, 1995, 1997; Simmons and Massey, 2012). To date, however, no study has directly compared the effects of feedback in the personal and social domains. Here, we hence 
examined the relative robustness of personal and social optimism biases with respect to (dis)confirming feedback information (Aim 5: Modifiability of personal and social optimism bias). Based on previous findings, we predicted limited malleability for both personal and social forms of optimism bias in light of single-shot feedback, with no specific hypothesis regarding their relative degrees of robustness. However, we expected personal and social biases to be present both before and after feedback administration.

We investigated our aims in an experiment, in which nonsoccer players and soccer players evaluated the probability of successful passes for different target players (self, rival of the self, an indirectly relevant in-group, an indirectly relevant out-group, an abstractly relevant in-group, and an abstractly relevant outgroup) facing different soccer scenarios. We also assessed our participants' degree of identification with the different in-group target characters, and part of our participants re-evaluated the probabilities after they had received feedback regarding the supposedly objective probability of a successful pass in each soccer scenario.

\section{Methods}

Participants. Participants comprised 160 (100 males; age: $M=24.1 ; S D=4.74)$ individuals, either undergraduate students or non-professional active soccer players (amateur league; training once or twice a week; competitive matches on the weekends). Recruitment made use of Internet advertisements and direct contacts with local soccer clubs. All participants were characterized by normal or corrected-to-normal vision and compensated with course credit or 15 Swiss Francs per hour. Study protocols had been approved by the local ethics committee and were in accordance with the Helsinki Declaration of Human Rights (World Medical Association, 1999). All participants gave written informed consent.

The participants underwent slightly different experimental procedures to allow for an adequate investigation of our study aims (encompassing aims that are not presented here: Physiological responses and gazing behavior were acquired for different subsets of our participants to gain deeper insight into the mechanisms underlying personal and social optimism biases, including potential differences between active soccer players and non-soccer players). Because of time restrictions, it was impossible to collect all relevant data from every participant. Sample 1 (non-soccer behavior) comprised individuals, who did not play soccer. Sample 2 (non-soccer physio) also consisted of non-soccer players, the sole difference with respect to sample 1 being that various somatovisceral and eye-tracking measures were additionally acquired. Sample 3 (soccer physio) underwent the same procedures as sample 2 but comprised active soccer players. Finally, sample 4 (non-soccer feedback) differed from the three other samples in that it (a) consisted of male and female participants; (b) faced only one in-group and one out-group condition (indirectly relevant to the participant); and (c) underwent a feedback manipulation. The feedback manipulation was included because we were interested in the malleability of the biases when people receive objective feedback. Sample sizes exceeded those of an earlier study with a highly similar paradigm (Aue et al., 2012). For an overview of sample characteristics, the samples' respective involvement in the investigation of our study aims, and the statistics applied see Table 1.

Task and procedure. The experiment was introduced as a study on foresighted thinking regarding more or less difficult situations in active soccer players vs. non-soccer players. The participants were told that the inclusion of different soccer player targets in

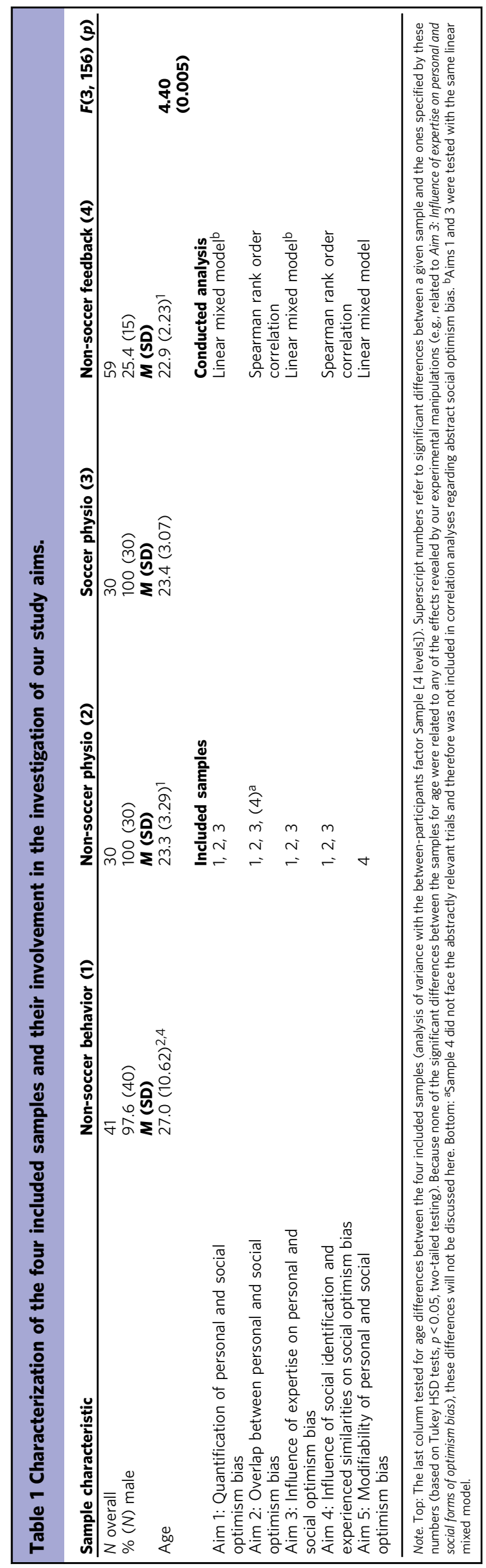




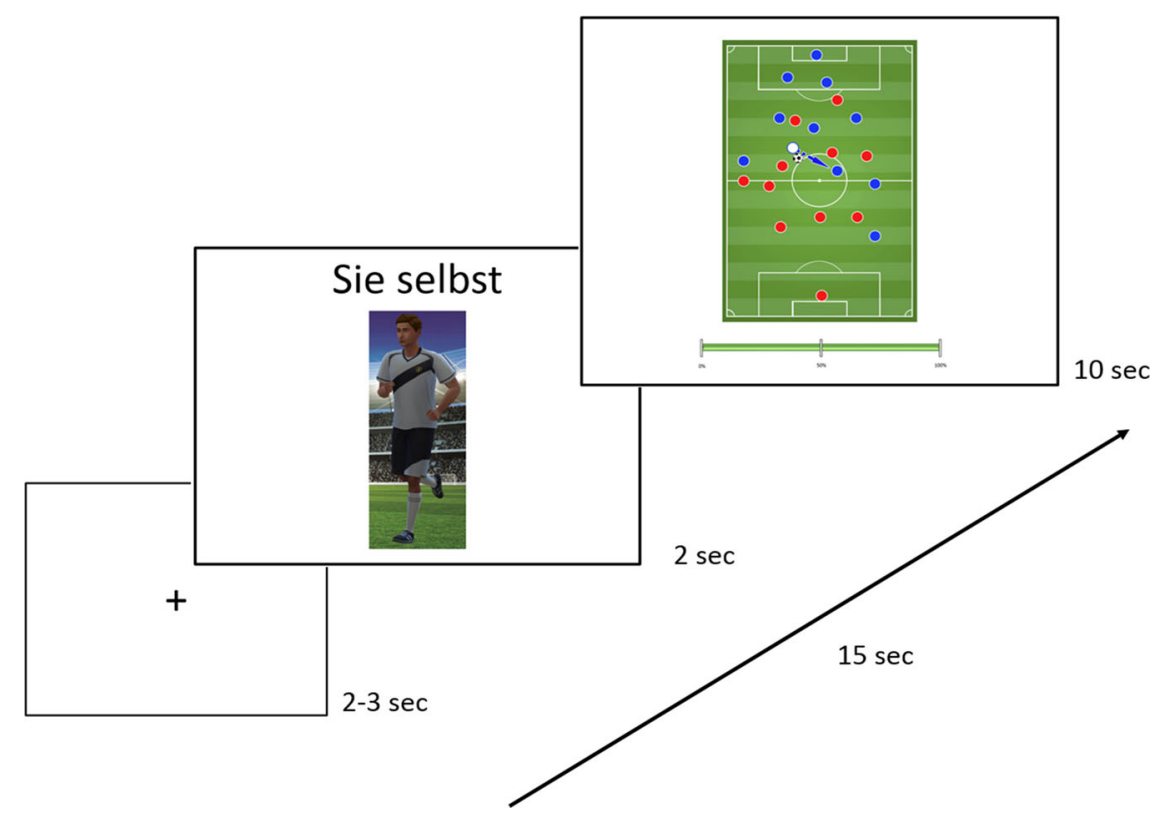

Fig. 1 Schematic representation of an experimental trial. "Sie selbst" indicates that the likelihood needs to be specified for the self.

the task served to decrease boredom. Their task was to estimate the probability that these target players would successfully pass a ball to a player of their own team or mark a goal. Participants faced 16 different soccer scenarios. These scenarios were represented by specific spatial constellations of teammates and opponents of a depicted target player. The same constellations were rated for six different social targets (detailed in the next paragraph). The target players were highlighted, and the direction of their future ball pass was depicted by an arrow (Fig. 1). Answers were given on a continuous visual analog scale that ranged from $0 \%$ (absolutely certain that pass will not be successful) to $100 \%$ (absolutely certain that a pass will be successful). The starting value of the selection slider was $50 \%$ and participants were able to shift the slider in each direction by pressing two different keys on a computer keyboard. The experiment was programmed with E-Prime 2.0 Professional (version 2.0.10.353; Psychology Software Tools, Pittsburgh, USA).

The soccer scenarios were presented in three different blocks (counterbalanced order). In one block, participants estimated the probability of successful passes for themselves and a personal rival. To establish rivalry, before starting the experiment, participants were faced with the scenario that a person with similar talent competed for their position in their current (imagined) soccer club. Contrasting these probability estimates yielded a measure of personal (i.e., directly relevant to the participants' goals) optimism bias. In a second block, participants estimated the same chances for an in-group that consisted of a fictitious first-league soccer team the participants hoped to join in the near future and thus most likely identified with. While our participants were not personally involved in those imagined scenarios, good performance of the potential future home team would increase the standing of this team, thereby having an indirect influence on the participants' own future possibilities. The corresponding out-group was the archrival of this team. Contrasting probability estimates for these two teams yielded our measure of indirect social optimism bias. To avoid connotations of actual players, the unknown in-group and out-group members in these indirectly relevant conditions were described as newly acquired players of similar talent. For the non-soccer behavior, non-soccer physio, and soccer physio samples, there was an additional block for social optimism bias. We included this third block because social optimism bias is observed even when an anticipated outcome does not have an influence on an individual's personal future (e.g., Aue et al., 2012; Babad, 1987, 1995). This added social optimism bias block assessed the anticipated performances of two social groups the participants would never be active parts of. Hence, the performances of these groups were abstractly relevant to the participants' goals. Specifically, in this third block, participants specified probability estimates for their preferred fictitious favorite international soccer team-a team they were supposedly an avid fan of-and the archrival of this team. The comparison of these two conditions thus yielded a measure of abstract social optimism bias.

Importantly, the players for whom the participants estimated the chances of successful passes were not in direct competition in any given trial and our participants were explicitly informed about this fact. This allowed the attribution of the observed effects to a specific social target. Color-coding of the jerseys (counterbalanced across participants) signaled whether a specific situation displayed concerned the self, the rival, an in-group member, or an out-group member. Abilities of the recipients of the social targets' passes as well as overall abilities of same and opponent teams were not specified. Consequently, assumed skills of these nontarget players should not have differed between the experimental conditions. In total, participants gave success estimates for six (in the non-soccer feedback sample: four) different players facing the same 16 soccer situations, totaling 96 (64 for the non-soccer feedback sample) experimental trials. At the end of the experiment, participants completed a series of questionnaires (see next section for a specification of the questionnaire of interest for the current research aims) and were debriefed.

The non-soccer feedback sample (sample 4) did not face the abstract optimism bias trials, but instead underwent a feedback manipulation. After having performed the first round of 64 trials, participants received feedback regarding the supposedly "objective" probability of success (based on estimations provided by active soccer players [soccer physio sample]) for each of the 16 soccer scenarios included in the study. They subsequently reevaluated all target player $\times$ soccer scenario combinations, which allowed us to verify to what extent the feedback had modified their initial expectancies. Feedback was given at a global levelwithout reference to the four social targets under investigation. 
Hence, the feedback related to different constellations of same and opponent team players on the soccer field rather than to abilities of the social targets.

Included questionnaire. Inclusion of Other in the Self Scale (IOS Scale; in-house development based on Aron et al., 1992). The IOS Scale was used to assess the perceived similarity (i.e., to what degree they are perceived as separate entities) between any set of two different target players. Related to Aim 4 (Influence of social identification and experienced similarities on social forms of optimism bias), participants evaluated the following similarities: (a) self/indirect in-group; and (b) self/abstract in-group. The IOS Scale consists of seven pairs of circles (here: one circle symbolling the self, the other circle symbolling the indirect or the abstract ingroup) that vary in degree of overlap. Participants chose the option that best described the degree of similarity they saw between the self and the two in-groups. Scoring varies from 1 (no overlap) to 7 (almost complete overlap).

\section{Data analysis and results}

Data selection. For the analyses regarding Aims 1, 3, and 4, the final sample consisted of 101 (100 male) participants (based on samples non-soccer behavior, non-soccer physio, and soccer physio; data from the non-soccer feedback sample were not considered because this sample did not face abstractly relevant trials). These participants exhibited, on average, $0.69 \%$ missing data. Analyses related to Aim 5 (modifiability of optimism bias) relied on the 59 ( 15 male) participants completing the feedback procedure (no missing data). Finally, analyses regarding Aim 2 (Overlap between personal and social forms of optimism bias) were based on participants of all four samples (correlation between personal and indirect social optimism bias) or all but the non-soccer feedback sample (correlations involving abstract social optimism bias). For better visualization refer to Table 1 .

Quantification of personal and social forms of optimism bias (Aim 1) including a test of the influence of expertise (Aim 3). To quantify the personal as well as the indirect and abstract social optimism biases, and simultaneously test for an influence of expertise, we used linear mixed modelling, as implemented in the GAMLj module in jamovi (The jamovi project (2020). jamovi. (Version 1.8.4) [Computer Software]. Retrieved from https:// www.jamovi.org.). To elucidate any interaction effects, we used the simple effects procedure as implemented in jamovi/GAMLj. The linear mixed model had two levels. Level 2 comprised the two fully crossed random effects variables Participants $(n=101)$ and Scenarios $(n=16)$. The participants' probability estimates for the six social target characters within each combination of participant and scenario constituted the level 1 outcome. Relevance (direct, indirect, abstract) and Character Valence (positive [self and ingroups], negative [rival and out-groups]) were level 1 categorical predictors, as they represented characteristics of the social target characters. Sample (non-soccer behavior, non-soccer physio, soccer physio) was a level 2 categorical predictor as it represented a characteristic of the participants.

In addition to random intercepts, we included random slopes of Relevance and of Character Valence for both random effects variables (Participants and Scenarios). Further adding random slopes for the Relevance $\times$ Character Valence interaction prevented convergence of the model. We, therefore, decided to keep only the main effect random slopes. All models were estimated with an unstructured covariance matrix (correlated random effects). The included random effect parameters were tested for significance using likelihood ratio test (LRT) comparisons with models that did not contain the respective effect in question. The results showed that both random slopes of Relevance and Character Valence were significant for the random effects variable Participants (LRT $(4)=157.60$ and LRT $(7)=150.59$, respectively, both $p s<0.001)$, indicating that the effects varied considerably across participants and this variation should be considered in the model. On the contrary, both random slopes were non-significant for the random effects variable Scenarios $(\operatorname{LRT}(4)=0.04, p=1.0$ and $\operatorname{LRT}(7)=4.41, p=0.732)$. To avoid overparameterization and related estimation problems we kept only the random slopes of Relevance and Character Valence for Participants in the final model. Because we focus on the fixed effects of our predictors, we refrain from reporting random effects (variances and covariances) parameter estimates in the following.

The model yielded a significant main effect of Relevance, $F(2$, $98.1)=5.821, p=0.007$. Successful passes were estimated to be (marginally) more likely in the directly relevant trials (i.e., self and rival combined; $M=60.4 \%$ ) compared with the indirectly (indirect in-group and out-group combined; $M=58.5 \%, p=0.017$ ) and with the abstractly (abstract in-group and out-group combined; $M=58.9 \%)$ relevant trials $(p=0.075)$. The difference between the indirectly and abstractly relevant trials did not reach significance ( $p=1.0$, all $p$ s according to post hoc Bonferroni tests). Furthermore, the main effect of Sample was marginally significant, $F$ (2, $98.0)=2.80, p=0.066(\mathrm{Ms}=56.6,57.8$, and 63.4, for non-soccer behavior, non-soccer physio, and soccer physio, respectively). Descriptively, soccer players gave higher probabilities than did non-soccer players, but none of the comparisons reached statistical significance ( $p s>0.074$ for all Bonferroni post hoc tests).

More important, the main effect of Character Valence was significant, $F(1,98.3)=14.07, p<0.001$. Consistent with the idea of an optimism bias, the participants' rated probability of successful passes was higher for positive characters (self and in-groups; $M=60.5 \%$ ) than for negative characters (rival and out-groups; $M=58.0 \%)$. Notably, there was a marginally significant interaction of Character Valence $\times$ Sample: $F(2,98.3)=2.94, p=0.058$ (Fig. 2), which points to the possible influence of prior experience on the overall magnitude (i.e., irrespective of type) of optimism bias displayed. In line with this idea, simple effects regarding the difference between positive and negative characters revealed that soccer expertise lowered the extent of optimism bias displayed, with a significant difference between estimates for positive and negative target characters in the non-soccer behavior sample, $F$ ( 1 , $98.2)=19.20, p<0.001$, and in the non-soccer physio sample, $F$ $(1,97.9)=3.34, p=0.035$ (one-tailed), but not in the soccer physio sample, $F(1,98.7)=0.39, p=0.266$ (one-tailed). Together, these results suggest that prior knowledge on the events to be rated does indeed influence the amount of bias displayed.

Finally, the interactions Relevance $\times$ Character Valence, Relevance $\times$ Sample, and Relevance $\times$ Character Valence $\times$ Sample failed to reach significance, $F(2,9211.6)=1.40, \quad F(4$, $98.0)=1.07$, and $F(4,9211.3)=1.35$, ps $>0.247$, respectively. Thus, the null hypothesis regarding the (difference in) magnitudes of the different optimism biases cannot be rejected.

\section{Overlap between personal and social forms of optimism bias} (Aim 2). In each participant, ratings available after missing exclusion were averaged for each of the six experimental conditions. To determine the degree of overlap between personal, indirect social, and abstract social optimism bias, we first calculated a personal optimism bias measure (average score selfaverage score rival), an indirect social optimism bias measure (average score indirect in-group-average score indirect outgroup), and an abstract social optimism bias measure (average score abstract in-group -average score abstract out-group) for each participant. Because the distributions of these bias scores 


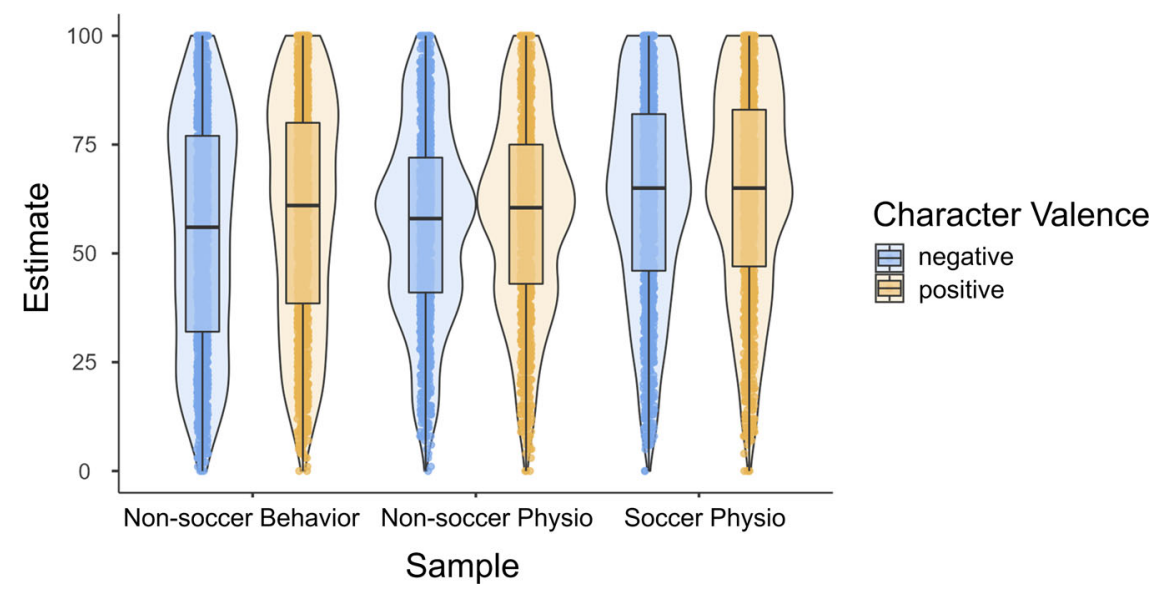

Fig. 2 Estimated probability of successful passes as a function of Character Valence and Sample. Violin and box plots depicting the significant interaction between Character Valence and Sample. Positive valence refers to self and in-groups, negative valence to rival and out-groups.

$\begin{array}{ll}\text { Rank } & \text { Rank } \\ \text { personal } & \text { indirect social } \\ \text { optimism bias } & \text { optimism bias }\end{array}$

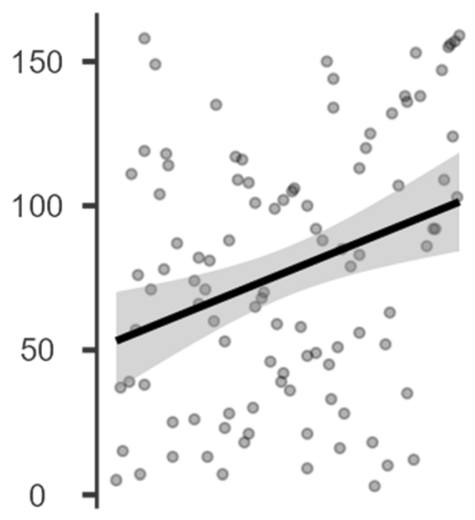

Rank
indirect social
optimism bias
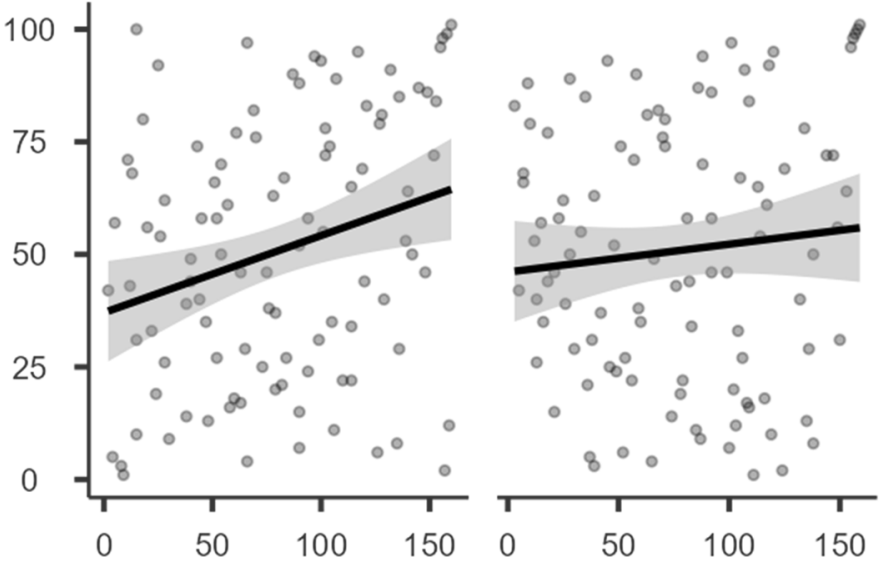

Fig. 3 Spearman correlations between the different forms of personal and social optimism bias. Personal optimism bias is based on the comparison of self vs. rival, indirect social optimism bias on the comparison fictitious potential future home team vs. its archrival, and abstract social optimism bias on the comparison fictitious favorite international soccer team vs. its archrival.

were not normal, we calculated Spearman rank-order correlation coefficients between the three biases to determine their overlap. The resulting correlations (Fig. 3) between personal optimism bias, on the one hand, and the two social optimism biases, on the other hand, were significant, but of relatively small size (personal and indirect social: $\rho(160)=0.303, p<0.001$; personal and abstract social: $\rho(101)=0.275, p=0.005$ (two-tailed; critical $\alpha$ for $n=3$ performed correlations: 0.0125$)$ ), signaling only a small proportion of shared variance. Somewhat surprisingly, the two social biases were not significantly associated, $\rho(101)=0.097$, $p=0.337$ (two-tailed), suggesting that these two forms of social bias refer to qualitatively different psychological concepts. 
Influence of social identification and experienced similarities on social forms of optimism bias (Aim 4). To test our hypothesis that social optimism bias was related to the degree of identification with the two in-groups, we calculated Spearman rank-order correlation coefficients between the social bias measures (see the previous section) and the degree of similarity indicated between the self and the in-group players as specified on the IOS Scale (see Table 2 for descriptive statistics). Our social identification hypothesis was not supported by the data: The correlation coefficient between the degree to which participants identified with the indirectly relevant in-group (self $\cap$ indirect ingroup), on the one hand, and the extent of indirectly relevant social optimism bias displayed, on the other hand, was not significant, $\rho(101)=0.015, p=0.443$ (one-tailed; critical $\alpha$ for $n=2$ performed correlations: 0.025 ). The same was observed when we tested the association between identification with the abstract ingroup (self $\cap$ abstract in-group) and abstractly relevant social optimism bias displayed, $\rho(101)=-0.056$ (significance is not specified for this latter correlation because the sign of the correlation coefficient points into the unexpected direction).

Modifiability of personal and social optimism bias (Aim 5). The non-soccer feedback sample evaluated the target players' probability of successful passes twice, with feedback given in-between. This repetition had been performed to investigate whether giving feedback would decrease personal and indirect social optimism bias. To test whether giving feedback influenced the participants' probability estimates, we again applied linear mixed modelling. Our fully crossed linear mixed model had two levels. At level 2 were situated random effects for Participants $(n=59)$ and Scenarios $(n=16$; twice repeated). The participants' probability estimates for the four social target characters (note the absence of abstract trials) within each combination of participant and scenario (considering its repetition) constituted the level 1 outcome. Relevance (direct, indirect), Character Valence (positive [self and in-group], negative [rival and out-group]), and Time (before feedback, after feedback) were level 1 categorical predictors, as they represented characteristics of the social target characters as well as a before-after component regarding these.

As for the first model, we included random slopes of the level-1 predictor main effects (thus for Relevance, Character Valence, and Time) for both random effects variables (Participants and Scenarios). Again, the model was estimated with an unstructured covariance matrix. The random slopes of Relevance, Character Valence, and Time were all significant for the random effects variable Participants (LRT $(4)=30.88$, LRT $(4)=42.66$, LRT $(4)=456.61$, respectively, all $p s<0.001)$, indicating that the effects varied considerably across participants and this variation should be considered in the model. For the random effects variable Scenarios, on the other hand, only the random slope of the Time effect was significant (LRT $(4)=57.50, p<0.001)$ while random slopes of Relevance and Character Valence were statistically irrelevant (LRT $(4)=0.65, p=0.958$, and LRT $(4)=1.73, p=0.785$, respectively). For the final model only the statistically relevant random slopes were included.

Only the main effects of Time, $F(1,57.2)=11.63, p<0.001$, and Character Valence, $F(1,58.0)=11.34, p=0.001$, achieved significance (Fig. 4). After having received feedback, participants envisaged higher probabilities of successful passes than before ( $M s=53.5 \%$ and $57.8 \%$, before and after feedback, respectively).

\section{Table 2 Overlap/similarity on the Inclusion of Others in the Self Scale.}

\begin{tabular}{lllllll} 
Overlap/similarity & M & SD & Min & Max & $\boldsymbol{F ( 2 , ~ 9 8 ) ~}$ & \multicolumn{1}{c}{0} \\
\hline Self $\cap$ indirect in-group & 5.2 & 1.99 & 1 & 7 & 0.52 & 0.595 \\
Self $\cap$ abstract in-group & 4.6 & 1.86 & 1 & 7 & 0.58 & 0.558 \\
\hline
\end{tabular}

Means $(M)$, standard deviations (SD), and minimum and maximum values displayed for the comparison of different players.

Note. $N=101$ (not assessed in sample 4 [non-soccer feedback sample]). $F$ and $p$ values refer to one-factorial analyses of variance (ANOVAs) testing for differences in the IOS Scale ratings between samples 1-3. These ANOVAs clearly show that identification did not differ between samples, thereby ruling out confounding effects of identification regarding Aim 3 (Influence of expertise on personal and social forms of optimism bias, see previous section) analyses.

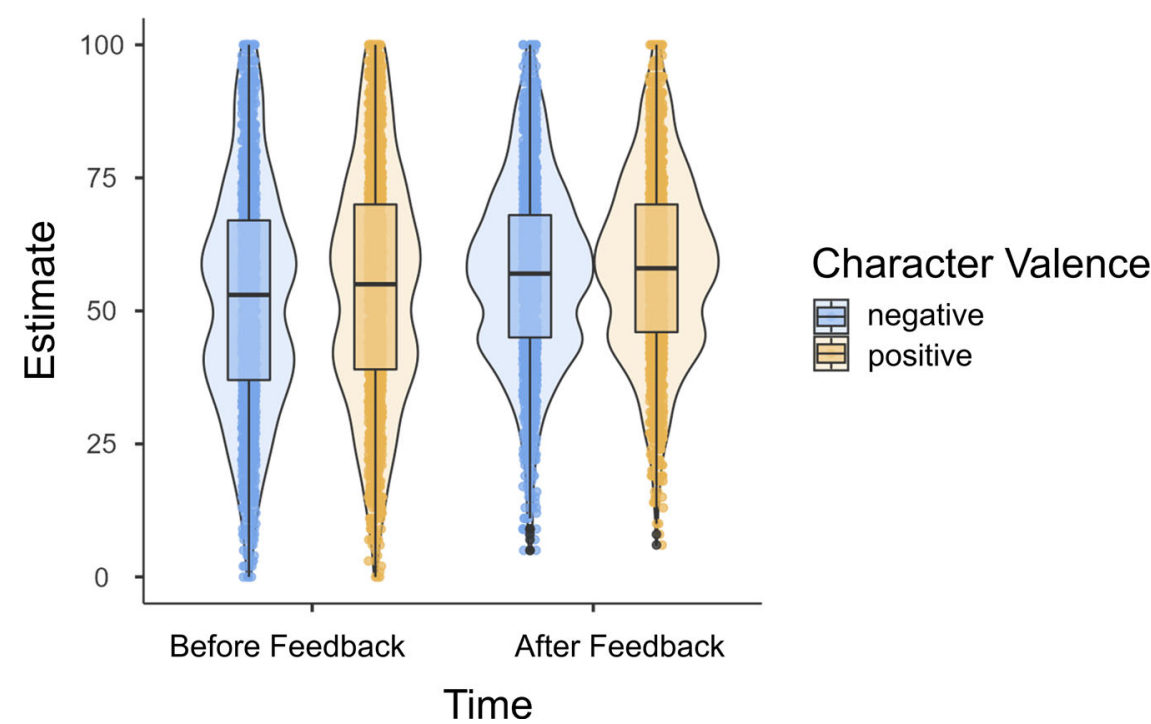

Fig. 4 Estimated probability of successful passes as a function of Character Valence and Time. Violin and box plots depicting the main effects of Character Valence and Time. Positive valence refers to self and indirect in-group, negative valence to rival and indirect out-group. The average feedback score given to the participants was $M=75.1 \%(S D=20.17 \%)$. 
This can be explained by the fact that, in most cases, the feedback given was higher than the initial estimate specified by our participants (because the feedback was based on the estimates of actual soccer players [soccer physio sample]). More important, participants in this sample displayed an optimism bias (main effect of Character Valence): Higher estimates were revealed in situations that concerned liked (self and in-group; $M=56.5 \%$, before and after feedback combined) rather than disliked (rival and out-group; $M=54.8 \%$ ) players.

Moreover, replicating the analyses conducted for Aim 1, the magnitude of optimism bias did not significantly differ between the personal and the social domain, interaction Relevance $x$ Character Valence, $F(1,7279.1)=0.06, p=0.813$, and interaction Relevance $\times$ Character Valence $\times$ Time, $F(1,7279.1)=0.61$, $p=0.433$. Furthermore, the interaction Character Valence $\times$ Time, $F(1,7279.1)=2.65, p=0.104$, did not reach statistical relevance either. One might argue that increasing the sample size might have resulted in a significant interaction. However, the simple effects analyses revealed significant differences between valence levels both before and after feedback, $F(1,107.9)=13.87$, $p<0.001$, and $F(1,107.9)=4.16, p=0.044$ (two-tailed), respectively, thus demonstrating that the feedback given in the current study was not able to eliminate the optimism bias. Neither the main effect of Relevance nor the interaction Relevance $\times$ Time reached significance, $F(1,58.0)=0.11, p=0.743$, and $F(1$, $7279.1)=2.84, p=0.092$, respectively.

\section{Discussion}

The current research compared personal optimism bias with two social optimism biases that varied in degree of relevance to the self. Specifically, we were interested in the relative magnitudes of and the overlap between the biases, in the influence of expertise and social identification, as well as in the biases' malleability. The following sections address these aims one after the other.

Quantification and overlap of personal and social forms of optimism bias (Aims 1 and 2). Participants in our studies were prone to personal optimism bias: They estimated their personal probability of performing a successful pass as higher than the rival's probability (direct relevance trials), replicating numerous earlier findings (e.g., Hoch, 1985; Lench and Ditto, 2008; Weinstein, 1980). Also consistent with prior observations (e.g., Aue et al., 2012; Dricu et al., 2018; Krizan et al., 2010; Simmons and Massey, 2012), social optimism bias was displayed, with higher estimates for the in-groups compared with the out-groups (for both indirect and abstract relevance trials). Moreover, in the current series of studies, there was no indication of marked differences between the magnitudes of personal and social optimism biases. Thus, the extent to which we are overoptimistic for ourselves does not per se differ from the degree of overoptimism exhibited in the social domain. Furthermore, optimism bias magnitude does not shrink with decreasing self-relevance. Notably, while the absence of significance cannot prove the absence of a difference, we argue that potential differences between personal and social biases in our studies must have been of negligible size.

From a comparison of the magnitudes of personal bias and the two social optimism biases, one might be tempted to conclude that all three reflect the same underlying mechanisms. Yet, our correlation analyses suggest them to be different, nonetheless. Concretely, there was only a weak association between personal optimism bias, on the one hand, and both social optimism biases, on the other hand. Even more surprising, there was no overlap between indirect and abstract social optimism bias, whereas both were supposed to rely on social identification processes.
The sole intended difference between the two social biases related to degree of relevance to the self. Specifically, we believed that the performance of the fictitious team that might be joined in due time would have at least an indirect impact on the participants' own future: Anticipating to join a good or a bad team might tell something about one's own capacities and one's to-be-envisaged soccer career. The most liked fictitious famous international team, on the contrary, did not imply a change in personal conditions and only involved the affective dimension (hence being only of abstract relevance). The present results, however, suggest that relevance to personal goals might not be the driving factor for social biases to arise. Consequently, potential moderators or mediators in the adoption of the different forms of social bias remain to be identified in future studies.

It is possible that imagining the potential future home team performing well is not necessarily unambiguously positive for any participant. Particularly those participants with low self-esteem might simultaneously feel scared because joining a good performing team implies stronger competition for positions within the team. Additionally, a very successful team might change its criteria for the selection of new players, thereby possibly preventing the participant to be chosen as a new member of the team (remember that our participants were not yet sure whether they would be able to join the potential future home team).

An alternative explanation for differences between indirect and abstract social optimism bias could be that people are used to identify with famous sports teams and therefore rather automatically adopt corresponding perspectives. The instructions in the current experiment may have specifically contributed to such automatic perspective taking in abstract social optimism bias. We told our participants that they were long-time fans of the given fictitious international team and their watching every soccer match of this team had long history dating back to childhood. By contrast, changing a reference group in any given area (i.e., changing one's soccer club; scenario for indirect social optimism bias) is generally rare and may thus relate to more effortful processing. It is also possible that differences between the two social biases arose because some of our participants adopted a more affective (abstract in-group) and others a more utilitarian (indirect in-group) stance. The processes mediating both social biases might hence strongly differ.

In sum, data of the current study suggest that people engage distinctly in the three types of bias investigated. This may also be related to individual differences in habits to increase positive emotionality: It is possible that our participants engaged preferably in one particular type of bias to uplevel their affect and did not need as much another type to maintain positive emotionality throughout the experiment. Related to this issue, we also tested for an effect of block order: Yet, participants who evaluated the personal (indirect/abstract social) trials first did not differ from those who faced those trials later in the experiment. Therefore, we can preclude that participants simply responded to the first pair of players presented to (up)regulate their affect (and subsequently maintained it throughout the experiment).

Overoptimism for both the self and social in-groups involves important social discrimination processes that may help to maintain a positive view of oneself (i.e., high self-esteem) and the world. Noteworthily, social trials, in contrast to personal trials, in the current study, did not contain any explicit notion of conflict: Introducing a social group as the archrival of the groups one identifies with or likes suffices to trigger social conflict that an individual needs to cope with. Correspondingly, earlier findings based on a similar paradigm measuring social optimism bias (Aue, 2014) has demonstrated that the anticipation of positive outcomes for the in-group and the anticipation of negative 
outcomes for a disliked out-group are associated with largely overlapping neural signatures.

In general, conflict-related aspects in optimism bias need greater in-depth investigation. To date, interpretation of overoptimism in the personal and social domains is mostly restricted to the individual or the social groups who receive the preferential treatment (self, in-group). Yet, there are also essential not-to-beneglected characteristics that are shared between the less favorably treated social targets (rival, out-group). Basically, the good performance of a personally disliked rival threatens selfesteem (e.g., Watanabe, 2019; see also Brunot and Juhel, 2012, for effects related to a comparison of self vs. a superior out-group) because it endangers the achievement of important personal goals (here: keeping the desired permanent position in a soccer team, self vs. rival trials). By contrast, good performance of an unknown -but still disliked-out-group member may threaten social selfesteem (e.g., Bardel et al., 2010; Smurda et al., 2006) by questioning the social in-group's goals (here: being among the best teams in the soccer league; both indirect and abstract ingroup vs. out-group trials).

Consequently, future studies in the area should closely examine whether the optimism bias displayed in the current study results from the upregulation of expectancies for self and in-groups and/ or the downregulation of expectancies for rival and out-groups. The additional inclusion of neutral target players might help to answer this question. That it is important to distinguish between those two origins is discussed in Ames' similarity contingency model of social inference (Ames, 2004). Correspondingly, behavioral and neural data show that people project selfattributes to close or similar others, but employ in stereotypes for dissimilar others (Cheng et al., 2010; Dricu et al., 2018; Krill and Platek, 2009).

Influence of expertise on personal and social forms of optimism bias (Aim 3). In the personal domain, optimism bias has been found to be reduced (but not totally eliminated) for increasing levels of experience with a to-be-evaluated scenario (e.g., Grezo, 2017; Helweg-Larsen and Shepperd, 2001; Weinstein, 1980). Here, we show that this effect can also be seen in the social domain. Soccer players, compared with novices in the field, are overall less prone to reveal optimism biases. Importantly, because IOS scores did not differ between samples 1-3 (Table 2), the reduced social optimism bias in the expert group cannot be attributed to diminished social identification or perception of similarities with the in-group target players. Together, these findings suggest that optimistic biases may be reduced by providing individuals with more insider knowledge on the situations under investigation. However, other research (e.g., Babad, 1987; Sharot et al., 2011) has shown that giving objective background information once does not sustainably reduce either personal or social optimism bias (see also discussion of Aim 5: Modifiability of personal and social forms of optimism bias). Therefore, decreasing overoptimism may necessitate greater personal involvement based on more extended and self-initiated occupations with critical situations (e.g. Barnoy et al., 2003).

Influence of social identification and experienced similarities with the target players on social forms of optimism bias (Aim 4). Social categorization leads to the experience of an out-group as a distinct entity that does not share many characteristics with the self (Tajfel and Turner, 1979). In contrast to the out-group, the in-group should be experienced as close to the self. Such differential social identification may lead a person to adopt the perspective "as if it were me" for in-group members (cf. Davis, 1983; Lamm et al., 2007, in empathy). Hence, the social optimism bias may rely on similar basic neurocognitive processes as the personal optimism bias does. Consistent with this view, strikingly similar processes have been hypothesized to be causally involved in both the maintenance of a positive image of the in-group (Tajfel and Turner, 1979) and in the maintenance of a positive self-image (Tesser, 2000).

The current study lends support to the idea that there is at least partial overlap between personal and social forms of optimism bias (see discussion related to Aim 2: Overlap between personal and social forms of optimism bias). Yet, such partial overlap between personal and social forms of overoptimism does not necessarily rely on the extent of identification with the social ingroups: Linking the participants' biases to their similarity ratings between the self and the in-groups on the IOS Scale did not reveal significant associations. These data converge with Brown's (2000) claims that strength of social identification does not automatically determine in-group favoritism. Alternatively, our participants may have engaged in the so-called belief in a just-world (Hafer and Begue, 2005; Lerner and Simmons, 1966), a phenomenon that describes an individual's conviction that good things happen to good people. Still another possibility is that basic valence priming (see Krizan and Windschitl, 2007) was at the origin of the observed effects.

Modifiability of personal and social forms of optimism bias (Aim 5). The feedback manipulation in our study was included to examine and compare the updating of personal and social optimism bias when people receive feedback about objective probabilities regarding the situations evaluated before. This question thus asked how malleable personal and social forms of optimism bias are in light of corrective feedback. Consistent with earlier research in the field (e.g., Chowdhury et al., 2014; Kuzmanovic et al., 2015, 2016; Marks and Baines, 2017; Sharot et al., 2011), we found that our participants-in spite of having received objective background information that underscored similar chances for all involved characters-continued to see their future soccer performance as better than their rival's. What is more, our data reveal that the indirect social optimism bias is, per se, equally robust.

Importantly, we emphasize that such restricted malleability of personal and social optimism bias is seen in experiments involving single-shot feedback. Based on our observations on the influence of expertise (see discussion for Aim 3: Influence of expertise on personal and social forms of optimism bias), we predict that more continuous and long-term confrontations with one's own overoptimism may lead an individual to ultimately employ in greater corrective actions, thereby reducing personal and social forms of optimism bias.

Prior findings in the personal domain (e.g., Chowdhury et al., 2014; Kuzmanovic et al., 2015, 2016; Marks and Baines, 2017; Sharot et al., 2011) demonstrated that individuals have difficulty in adapting their expectancies regarding important personal future life events when the information presented suggests the need to adapt expectancies into the pessimistic direction (bad news; e.g., average probability to incur cancer is higher than initially predicted for oneself). If, on the other hand, the information presented suggested the need to adapt expectancies into the optimistic direction (good news; e.g., average probability to incur cancer is lower than initially predicted for oneself), individuals readily grasp this information and update their expectancies accordingly.

Along these lines, one would further aim at investigating whether such updating asymmetries are also seen in the social area. However, the feedback participants (sample 4) received was almost exclusively "better" than expected because it was 
based on the estimations given by the active soccer players (sample 3). The traditional updating analyses in literature, therefore, were not applicable here. Future studies should ensure that participants receive both good and bad news. We predict outcome expectancies for an in-group to be more resistant to bad news than to good news and the reverse effect for a disliked out-group.

\section{Conclusions and future directions}

Research has shown substantial differences between optimistic biases arising in positive and negative situations (e.g., Dricu et al., 2018; Hoorens, 1996; Kress and Aue, 2019). For instance, self-enhancement might be the underlying mechanism for biases related to positive, but not negative, future events (Hoorens, 1996). In the current study, participants specifically imagined positive outcomes (i.e., scenarios were phrased positively, emphasizing the positive outcome [successful passes]). Future studies hence need to investigate magnitudes and overlap of personal and social optimism biases with respect to undesirable scenarios. In addition, potential gender and cultural differences in personal and social optimism biases need to be addressed.

Furthermore, although behavioral research on social optimism bias is in itself impressive, the neurocognitive mechanisms underlying this phenomenon are only poorly understood. To date, only few studies have been conducted in the area (Aue et al., 2012; Dricu et al., 2020; Moser et al., $2021,2020)$. To further investigate and interpret potential differences between personal and social optimism bias, we are engaged with collecting and analyzing somatovisceral responses as well as eye tracking data with the same experimental paradigm. Moreover, the missing overlap of the two social biases included in the current series of studies (Aim 2: Overlap between personal and social forms of optimism bias) suggests the existence of qualitatively different forms of social optimism bias (see also Dricu et al., 2018, 2020). Examinations of such social biases, therefore, may want to specifically address divergent underlying neurocognitive mechanisms.

Identification of the neurocognitive mechanisms at the basis of social overoptimism may provide important starting points for the modification of social biases that turn out problematic for the individual or the society. Because biases in social expectancies affect social interactions, the results of studies in this project may have meaningful implications for the development of social conflict reduction strategies. For instance, in some individuals, it may be advisable to promote social optimism bias (e.g., regarding the pursuit of important goals in a team sport [winning a match/gold medal]). If people think that a desired outcome is unlikely because they do not believe in others or the group as a whole, they will decrease individual efforts with possibly contagious effects on other members in their team (Wergin et al., 2018), thus making the outcome even less likely.

Notably, optimism bias often also comes with a denigration of rivals or social out-groups (Dricu et al., 2018). To reduce discriminative behavior, (social) optimism biases, in some cases, therefore, need to be actively reduced. Because people are generally resistant to decrease their optimistic biases, the identification of more efficient means to the reduction of unfavorable biases turns out essential. Our findings on the effects of expertise vs. single-shot feedback suggest that longterm rather than short-term campaigns may succeed in this endeavor. It may also be required that people have first-hand experience with the events that they are anticipating for others.
In addition, our data revealed the possible existence of different routes to foster well-being and life satisfaction, namely by increasing either personal optimism, or specific forms of social optimism. This observation may inspire therapeutical approaches for the treatment of depression. The inability to increase personal optimism does not necessarily go hand in hand with the inability to increase social optimism. Because self-esteem and zest for life may also be increased by social identification processes, individually tailored treatments should start to target boosting of social optimism in various domains that are of relevance to the patient.

Finally, future research may want to address similar biases in other domains (e.g., interpretation, memory, or attention) and look for commonalities across those domains. For instance, bidirectional causal influences between optimism bias and positive attention bias have been demonstrated in the personal domain (see Kress and Aue, 2017, 2019; Kress et al., 2018; Singh et al., 2020). In the social domain such causal associations await to be addressed. Social biases have been reported for various constructs, including attributions (Hewstone, 1990; Hugenberg and Bodenhausen, 2003) and memory (Howard and Rothbart, 1980; Jackson and Rose, 2013). These examples show that social comparison processes are strong motivational forces in the development of information processing biases-biases that might interact and function as an upward spiral of social positivity (Fredrickson and Joiner, 2018).

\section{Data availability}

Under the Swiss guidelines of data protection (Ordinance HFV Art. 5), the data sets generated and analyzed during the current study can be made available from the corresponding author on a case-by-case basis.

Received: 8 January 2021; Accepted: 28 September 2021; Published online: 14 October 2021

\section{References}

Abrams D, Hogg MA (2004) Metatheory: lessons from social identity research. Pers Soc Psychol Rev 8(2):98-106

Abrams D, Hogg MA (2010) Social identity and self-categorization. In: Dovidio JF, Hewstone M, Glick P, Esses VM (eds.) The SAGE handbook of prejudice, stereotyping and discrimination. Sage, London, UK, pp. 179-193

Ames DR (2004) Strategies for social inference: a similarity contingency model of projection and stereotyping in attribute prevalence estimates. J Pers Soc Psychol 87(5):573-585

Aron A, Aron EN, Smollan D (1992) Inclusion of other in the self scale and the structure of interpersonal closeness. J Pers Soc Psychol 63(4):596-612

World Medical Association (1999) Proposed revision of the declaration of Helsinki. Bull Med Ethics 150:18-22

Aue $\mathrm{T}$ (2014) I feel good whether my friends win or my foes lose: brain mechanisms underlying feeling similarity. Neuropsychologia 60:159-167

Aue T, Nusbaum HC, Cacioppo JT (2012) Neural correlates of wishful thinking. Soc Cogn Affect Neurosci 7(8):991-1000

Babad E (1987) Wishful thinking and objectivity among sports fans. Soc Behav 2(4):231-240

Babad E (1995) Can accurate knowledge reduce wishful thinking in voters' predictions of election outcomes? J Psychol 129(3):285-300

Babad E (1997) Wishful thinking among voters: motivational and cognitive influences. Int J Public Opin Res 9(2):105-125

Bardel MH, Fontayne P, Colombel F, Schiphof L (2010) Effects of match result and social comparison on sport state self-esteem fluctuations. Psychol Sport Exerc 11(3):171-176

Barnoy S, Bar-Tal Y, Treister L (2003) Effect of unrealistic optimism, perceived control over disease, and experience with female cancer on behavioral intentions of Israeli women to undergo screening tests. Cancer Nurs 26(5):363-369

Boen F, Vanbeselaere N, Pandelaere M, Dewitte S, Duriez B, Snauwaert B, Van Avermaet E (2002) Politics and basking-in-reflected-glory: a field study in Flanders. Basic Appl Soc Psych 24(3):205-214 
Brunot S, Juhel J (2012) Social and temporal comparisons, self-esteem, and jobsearch activity among long-term unemployed people. Annee Psychol 112(2):197-226

Campbell J, Greenauer N, Macaluso K, End C (2007) Unrealistic optimism in internet events. Comput Hum Behav 23(3):1273-1284

Cheng YW, Chen CY, Lin CP, Chou KH, Decety J (2010) Love hurts: an fMRI study. NeuroImage 51(2):923-929

Cho H, Lee JS, Chung S (2010) Optimistic bias about online privacy risks: Testing the moderating effects of perceived controllability and prior experience. Comput Hum Behav 26(5):987-995

Chowdhury R, Sharot T, Wolfe T, Duzel E, Dolan RJ (2014) Optimistic update bias increases in older age. Psychol Med 44(9):2003-2012

Cialdini RB, Borden RJ, Thorne A, Walker MR, Freeman S, Sloan LR (1976) Basking in reflected glory-3 (football) field studies. J Pers Soc Psychol 34(3):366-375

Davis MH (1983) Measuring individual differences in empathy: evidence for a multidimensional approach. J Pers Soc Psychol 44(1):113-126

Dricu M, Bührer S, Hesse F, Eder C, Posada A, Aue T (2018) Warmth and competence predict overoptimistic beliefs for out-group but not in-group members. PLoS ONE 13(11):e0207670

Dricu M, Kress L, Aue T (2020) The neurophysiological basis of optimism bias. In: Aue T, Okon-Singer $\mathrm{H}$ (eds) Cognitive biases in health and psychiatric disorders: neurophysiological foundations. Elsevier, San Diego, pp. 41-70

Dricu M, Schüpbach L, Bristle M, Wiest R, Moser DA, Aue T (2020) Group membership dictates the neural correlates of social optimism biases. Sci Rep 10(1):1-17

Fox E (2012) Rainy brain, sunny brain: The new science of optimism and pessimism. William Heinemann, London, UK

Fredrickson BL, Joiner T (2018) Reflections on positive emotions and upward spirals. Perspect Psychol Sci 13(2):194-199

Garrett N, Sharot T, Faulkner P, Korn CW, Roiser JP, Dolan RJ (2014) Losing the rose tinted glasses: neural substrates of unbiased belief updating in depression. Front Hum Neurosci 8:639

Granberg D, Holmberg S (1988) The political system matters: Social psychology and voting behavior in Sweden and the United States. Cambridge University Press, Cambridge, UK

Grezo M (2017) We expect stocks to rise, but we do not know when and which ones: excessive optimism in predicting future stock indices returns. Stud Psychol 59:113-126

Hafer CL, Begue L (2005) Experimental research on just-world theory: problems, developments, and future challenges. Psychol Bull 131(1):128-167

Helweg-Larsen M, Shepperd JA (2001) Do moderators of the optimistic bias affect personal or target risk estimates? A review of the literature. Pers Soc Psychol Rev 5(1):74-95

Hewstone M (1990) The "ultimate attribution error"? A review of the literature on intergroup causal attribution. Eur J Soc Psychol 20(4):311-335

Hoch SJ (1985) Counterfactual reasoning and accuracy in predicting personal events. J Exp Psychol Learn Mem Cogn 11(4):719-731

Hogg MA, Abrams D (1990) Social motivation, self-esteem, and social identity. In: Abrams D, Hogg MA (eds.) Social identity theory: constructive and critical advances. Springer, New York, NY, pp. 28-47

Hollander B (2004) People think like me: religion and wishful thinking in the 2000 US Presidential election. J Media Relig 3(4):187-197

Hoorens V (1996) Self-favoring biases for positive and negative characteristics: Independent phenomena? J Soc Clin Psychol 15(1):53-67

Howard JW, Rothbart M (1980) Social categorization and memory for in-group and out-group behavior. J Pers Soc Psychol 38(2):301-310

Hugenberg K, Bodenhausen GV (2003) Facing prejudice: implicit prejudice and the perception of facial threat. Psychol Sci 14(6):640-643

Jackson JW, Rose J (2013) The stereotype consistency effect is moderated by group membership and trait valence. J Soc Psychol 153(1):51-61

Korn CW, Sharot T, Walter H, Heekeren HR, Dolan RJ (2014) Depression is related to an absence of optimistically biased belief updating about future life events. Psychol Med 44(3):579-592

Kress L, Aue T (2017) The link between optimism bias and attention bias: a neurocognitive perspective. Neurosci Biobehav Rev 80:688-702

Kress L, Aue T (2019) Learning to look at the bright side of life: attention bias modification training enhances optimism bias. Front Hum Neurosci 13:222

Kress L, Bristle M, Aue T (2018) Seeing through rose-colored glasses: how optimistic expectancies guide visual attention. PLoS ONE 13(2):e0193311

Krill A, Platek S (2009) In-group and out-group membership mediates anterior cingulate activation to social exclusion. Front Evol Neurosci 1:1

Krizan Z, Miller JC, Johar O (2010) Wishful thinking in the 2008 US Presidential election. Psychol Sci 21(1):140-146

Krizan Z, Windschitl PD (2007) The influence of outcome desirability on optimism. Psychol Bull 133(1):95-121
Kuzmanovic B, Jefferson A, Vogeley K (2015) Self-specific optimism bias in belief updating is associated with high trait optimism. J Behav Decis Mak 28(3):281-293

Kuzmanovic B, Jefferson A, Vogeley K (2016) The role of the neural reward circuitry in self-referential optimistic belief updates. NeuroImage 133:151-162

Lamm C, Batson CD, Decety J (2007) The neural substrate of human empathy: effects of perspective-taking and cognitive appraisal. J Cogn Neurosci 19(1):42-58

Lench HC, Ditto PH (2008) Automatic optimism: biased use of base rate information for positive and negative events. J Exp Soc Psychol 44(3):631-639

Lerner MJ, Simmons CH (1966) Observer's reaction to the "innocent victim": compassion or rejection? J Pers Soc Psychol 4(2):203-210

Love BC, Kopec L, Guest O (2015) Optimism bias in fans and sports reporters. PLoS ONE 10(9):e0137685

Marks J, Baines S (2017) Optimistic belief updating despite inclusion of positive events. Learn Motiv 58:88-101

McGuire WJ (1960) A syllogistic analysis of cognitive relationships. In: Rosenberg MJ, Hovland CI, McGuire WJ, Abelson RP, Brehm JW (eds.) Attitude organization and change. Yale University Press, New Haven, pp. 65-111

McNally RJ (1995) Automaticity and the anxiety disorders. Behav Res Ther 33(7):747-754

Moser DA, Dricu M, Kotikalapudi R, Doucet GE, Aue T (2021) Reduced network integration in default mode and executive networks is associated with social and personal optimism biases. Hum Brain Mapp 42(9):2893-2906

Moser DA, Dricu M, Wiest R, Schüpbach L, Aue T (2020) Social optimism biases are associated with cortical thickness. Soc Cogn Affect Neurosci 15(7):745-754

Scheier MF, Carver CS (1992) Effects of optimism on psychological and physical well-being: theoretical overview and empirical update. Cognit Ther Res 16(2):201-228

Seligman MEP, Csikszentmihalyi M (2000) Positive psychology—an introduction. Am Psychol 55(1):5-14

Sharot T, Korn CW, Dolan RJ (2011) How unrealistic optimism is maintained in the face of reality. Nat Neurosci 14(11):1475-1479

Shepperd JA, Waters EA, Weinstein ND, Klein WMP (2015) A primer on unrealistic optimism. Curr Dir Psychol Sci 24(3):232-237

Simmons JP, Massey C (2012) Is optimism real? J Exp Psychol Gen 141(4):630-634

Sing L, Schüpbach L, Moser DA, Wiest R, Hermans EJ, Aue T (2020) The effect of optimistic expectancies on attention bias: Neural and behavioral correlates. Sci Rep 10(1):6495.

Smurda JD, Wittig MA, Gokalp G (2006) Effects of threat to a valued social identity on implicit self-esteem and discrimination. Group Process Intergroup Relat 9(2):181-197

Tajfel H, Billig MG, Bundy RP, Flament C (1971) Social categorization and intergroup behaviour. Eur J Soc Psychol 1(2):149-177

Tajfel H, Turner JC (1979) An integrative theory of intergroup conflict. In: Austin WG, Worchel S (eds) The social psychology of intergroup relations. Brooks/ Cole, Monterey, pp. 33-47

Tajfel H, Turner JC (1986) The social identity theory of intergroup behaviour. In: Worchel S, Austin WG (eds.) Psychology of intergroup relations. NelsonHall, Chicago, pp. 7-24

Tesser A (2000) On the confluence of self-esteem maintenance mechanisms. Pers Soc Psychol Rev 4(4):290-299

Turner JC, Onorato RS (1999) Social identity, personality, and the self concept: a self categorization perspective. In: Tyler TR, Kramer RM, John OP (eds.) The psychology of the social self. Erlbaum, Mahwah, pp. 11-46

Verkuyten M (2007) Ethnic in-group favoritism among minority and majority groups: testing the self-esteem hypothesis among preadolescents. J Appl Soc Psychol 37(3):486-500

Watanabe H (2019) Sharing schadenfreude and late adolescents' self-esteem: does sharing schadenfreude of a deserved misfortune enhance self-esteem? Int J Adolesc Youth 24(4):438-446

Weinstein ND (1980) Unrealistic optimism about future life events. J Pers Soc Psychol 39(5):806-820

Weinstein ND, Klein WM (1995) Resistance of personal risk perceptions to debiasing interventions. Health Psychol 14(2):132-140

Wergin VV, Zimanyi Z, Mesagno C, Beckmann J (2018) When suddenly nothing works anymore within a team-causes of collective sport team collapse. Front Psychol 9:2115.

Windschitl PD, Stuart JOR (2015) Optimism biases. In: Keren G, Wu G (eds.) The Wiley Blackwell handbook of judgment and decision making, 2nd edn. John Wiley and Sons, Ltd, Chichester, UK, pp. 431-455

Zetsche U, Rief W, Exner C (2015) Individuals with OCD lack unrealistic optimism bias in threat estimation. Behav Ther 46(4):510-520 


\section{Acknowledgements}

This research was supported by grants PP00P1_150492 and PP00P1_183709 of the Swiss National Science Foundation (http://www.snf.ch/en/Pages/default.aspx) to Tatjana Aue. The funders had no role in study design, data collection and analysis, decision to publish, or preparation of the manuscript.

\section{Competing interests}

The authors declare no competing interests.

\section{Additional information}

Correspondence and requests for materials should be addressed to Tatjana Aue.

Reprints and permission information is available at http://www.nature.com/reprints

Publisher's note Springer Nature remains neutral with regard to jurisdictional claims in published maps and institutional affiliations. (c) (i) Open Access This article is licensed under a Creative Commons Attribution 4.0 International License, which permits use, sharing, adaptation, distribution and reproduction in any medium or format, as long as you give appropriate credit to the original author(s) and the source, provide a link to the Creative Commons license, and indicate if changes were made. The images or other third party material in this article are included in the article's Creative Commons license, unless indicated otherwise in a credit line to the material. If material is not included in the article's Creative Commons license and your intended use is not permitted by statutory regulation or exceeds the permitted use, you will need to obtain permission directly from the copyright holder. To view a copy of this license, visit http://creativecommons.org/ licenses/by/4.0/.

(C) The Author(s) 2021 